\title{
64. Suggested Programming Project
}

For each of the following programs - produce structure diagrams, a list of paragraph names to be used, a written version of the program before testing, a test plan and log, comments on any changes that were necessary to cure logic errors and a printout of the final correct version of the program

A college needs to store details of students' exam results on disk and produce a report showing passes and fails. Produce the following.

Program 1 - which allows the user to key in a record for each subject passed as follows:

subject code

subject name

4 digits (including modulus 11 check-digit)

student surname

20 characters

student initials

15 characters

2 characters

student course code $\quad 6$ character $(1$ alphabetic +5 numeric)

result

1 character $(\mathrm{P}$ or $\mathrm{F})$

When you run the program, type the information in so that it is in order of subject code and within this in alphabetical order of student name.

Program 2 - which will read the file produced by Program 1 and validate the following fields:

subject code

student course code

result

This program should produce a file containing all the valid records and a printed report showing each record that contains an error (separate page for each record) with an indication of which fields are invalid.

Program 3 - which will read the valid file produced by Program 2 and produce a report set out as follows:

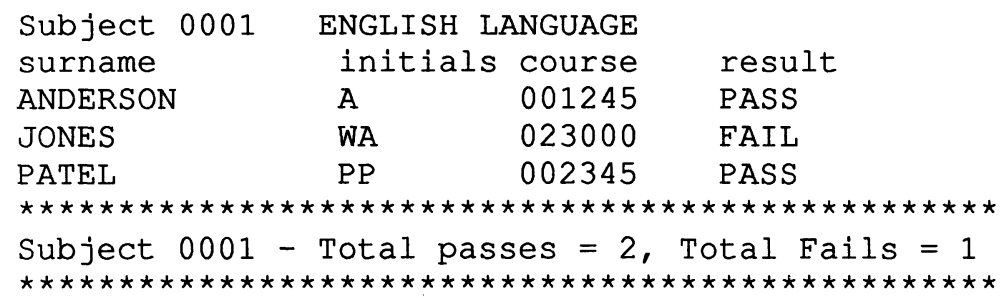

There should be a fresh page for each subject and in addition every 30 lines.

At the end of the report (on a fresh page) there should be totals for the number who took the exam, the number who passed and the number who failed. 